\title{
Lacrimal Mucocele
}

National Cancer Institute

\section{Source}

National Cancer Institute. Lacrimal Mucocele. NCI Thesaurus. Code C98968.

A congenital or acquired mucocele that develops in the lacrimal sac. It is usually the result of nasolacrimal duct abnormalities or obstruction. 